\title{
Outcome of monochorionic twins conceived by assisted reproduction
}

\author{
Teresinha Simões, M.D., M.Sc., ${ }^{a}$ Alexandra Queirós, M.D.,, Ana Teresa Marujo, M.D., ${ }^{a}$ \\ Sandra Valdoleiros, M.D., ${ }^{a}$ Patricia Silva, M.D., ${ }^{a}$ and Isaac Blickstein, M.D. ${ }^{b}$ \\ a Department of Maternal-Fetal Medicine Maternity Hospital, Dr. Alfredo da Costa, Centro Hospitalar Lisboa Central and \\ Universidade Nova de Lisboa, Lisbon, Portugal; and ${ }^{\mathrm{b}}$ Obstetrics and Gynecology, Kaplan Medical Center, Rehovot, and the \\ Hadassah-Hebrew University School of Medicine, Jerusalem, Israel
}

Objective: To evaluate monochorionic twins conceived by assisted reproductive technology (ART).

Design: We compared perinatal outcomes of monochorionic twins conceived by ART with their dichorionic counterparts and with spontaneous monochorionic twins.

Setting: Referral center.

Patient(s): Mothers to monochorionic and dichorionic twins conceived by ART and spontaneous monochorionic twins.

Intervention(s): None.

Main Outcome Measure(s): Maternal characteristics, pregnancy complications, and perinatal outcomes.

Result(s): Monochorionic twin pregnancies $(n=25)$ comprise $7.2 \%$ of all ART twins and 4.9\% of all monochorionic twins in this data set. Monochorionic pairs have a significantly worse outcome compared with dichorionic sets in terms of lower gestational age and birth weight. ART appears to increase the already high risk of monochorionicity compared with spontaneous conception: odds ratio (OR), 2.9 (1.1-7.3) for preterm birth at $<32$ weeks and OR, 5.9 (2.5-1.49) for birth weight $<1,500 \mathrm{~g}$.

Conclusion(s): Monochorionic twins after ART are at increased risk of adverse perinatal outcomes compared with spontaneous monochorionic twins and with dichorionic twins conceived by ART. (Fertil Steril ${ }^{\circledR}$ 2015;104:629-32. (C)2015 by American Society for Reproductive Medicine.) Key Words: Monochorionic twins, assisted reproduction, pregnancy complications

Discuss: You can discuss this article with its authors and with other ASRM members at http:// fertstertforum.com/simoest-monochorionic-twins-art/

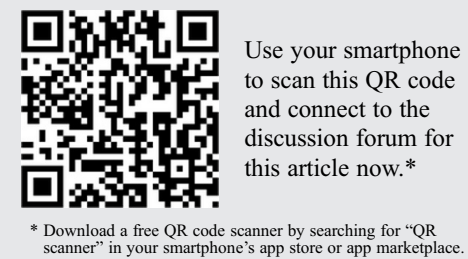

$\mathbf{T}$ he monozygotic twinning rate increases after all methods of infertility treatment (1-3). In contrast to ovulation induction techniques, where the number of resulting embryos is practically uncontrolled, assisted reproductive technology (ART, i.e., all methods of IVF and ET) offers a reduced risk of multiple pregnancies with single ETs. Yet the potential risk of zygotic splitting after ART has become clear. In a recent study from the United States, Gee et al. found that in $0.5 \%$ of twin, $29 \%$ of triplet, and in $64 \%$ of quadruplet births there were fewer fresh embryos transferred than the number of births, indicating that monozygotic twinning might be related to iatrogenic plurality (4). This method, like many others, does not count the frequency of monochorionic twins-the subset of monozygotic twins with the highest risk of fetal morbidity and mortality (5).

The outcomes of monochorionic twins conceived by ART have not been extensively studied, and the two available studies yield conflicting results. Mascarenhas et al. (6) evaluated monochorionic twins conceived by ART and concluded that these pregnancies have increased fetal loss when compared with dichorionic pregnancies. In contrast, Ghalili et al. (7) did

Received May 17, 2015; revised May 30, 2015; accepted June 2, 2015; published online June 17, 2015. T.S. has nothing to disclose. A.Q. has nothing to disclose. A.T.M. has nothing to disclose. S.V. has nothing to disclose. P.S. has nothing to disclose. I.B. has nothing to disclose.

Reprint requests: Isaac Blickstein, M.D., Department of Obstetrics and Gynecology, Kaplan Medical Center, 76100 Rehovot, Israel (E-mail: blick@netvision.net.il).

Fertility and Sterility® Vol. 104, No. 3, September 2015 0015-0282/\$36.00

not find significant differences between spontaneous and ART monochorionic pregnancies. In light of this ambiguity we sought to compare the perinatal outcomes of monochorionic twins conceived by ART with their dichorionic counterparts and with spontaneous monochorionic twins.

\section{MATERIAL AND METHODS}

We evaluated twin pregnancies, followed and delivered $\geq 24$ weeks' gestation at the Maternity Hospital Dr. Alfredo da Costa, Lisbon, Portugal, during the period September 1, 1994, through December 31, 2014. Our hospital is a tertiary perinatal center that cares for the Lisbon area and serves as a referral center for the south of Portugal. Information about pregnancy and delivery was registered prospectively on a preset form and subsequently entered into a computerized system. We excluded twin 
gestations that were delivered only and were not followed at our service.

Monochorionicity was established by standard ultrasound criteria confirmed by postpartum placental clinical and pathological examination; gestational age was calculated from the last menstrual period confirmed by first trimester sonography. In this study there were no monoamniotic sets after ART, hence we excluded monoamniotic sets from the controls as well. Indicated preterm deliveries were carried out on the basis of maternal and/or fetal conditions. We offered weekly to twice weekly follow-up including sonographic fetal biophysical profile and nonstress fetal heart rate testing, biweekly fetal growth assessment, and Doppler velocimetry of the umbilical and middle cerebral arteries as indicated.

In otherwise normally progressing gestations, we offered, after detailed counseling, elective deliveries at 36-37 completed weeks of gestation. We compared the perinatal outcomes of monochorionic-diamniotic twins conceived by ART (all methods of IVF and ET) with their dichorionic counterparts and with spontaneous (no treatment) monochorionic twins. Because of the rarity of the condition, we did not subdivide cases by the nature of embryo (fresh or thawed). We considered the following variables: maternal age and parity; maternal complications such as premature contractions ( $<34$ weeks of gestation); hypertensive disorders (preeclampsia, pregnancy-induced hypertension, and chronic hypertension); gestational diabetes; preterm rupture of membranes at $<34$ weeks of gestation; mode of delivery; gestational age at birth; birth weight; frequency of births at less than 32,
32-35, and 36 or more weeks (reason for preterm birth was not specified); birth weight less than 1,500 g, 1,500-2,499, and $2,500 \mathrm{~g}$ or more and birth weight discordance of $>25 \%$ (intertwin birth weight difference expressed as percentage of the heavier twin, calculated per total number of pregnancies); frequency of twin-twin transfusion syndrome (TTTS); frequency of Apgar scores $<7$ at 5 minutes; major malformations (excluding stillbirths, calculated per total number of fetuses); and early ( $<7$ days of life) neonatal death (calculated per total number of fetuses).

We compared continuous data by using two-tailed Student's $t$ test, and categorical data by two-tailed $\chi^{2}$ or Fisher's exact tests. We used SPSS, version 17, for statistical analyses. $P<.05$ was considered statistically significant for continuous data. We calculated the odds ratio (OR) and 95\% confidence interval (CI) for categorical data. Because of anonymous data collection, the study was exempt from approval by the local Institutional Review Board.

\section{RESULTS}

The data included 25 monochorionic twin pregnancies (50 twins) and 320 dichorionic twin pregnancies (640 twins) conceived by ART as well as 483 (966 twins) spontaneous monochorionic twins. Thus monochorionic twins comprise $7.2 \%$ of all ART twins, and monochorionic twins after ART comprise $4.9 \%$ of all monochorionic twins. Table 1 shows the comparison between monochorionic twins conceived by ART and spontaneous monochorionic twins. The data indicate that ART mothers were significantly older

\section{TABLE 1}

Comparison between monochorionic twins conceived by ART and spontaneous monochorionic twins.

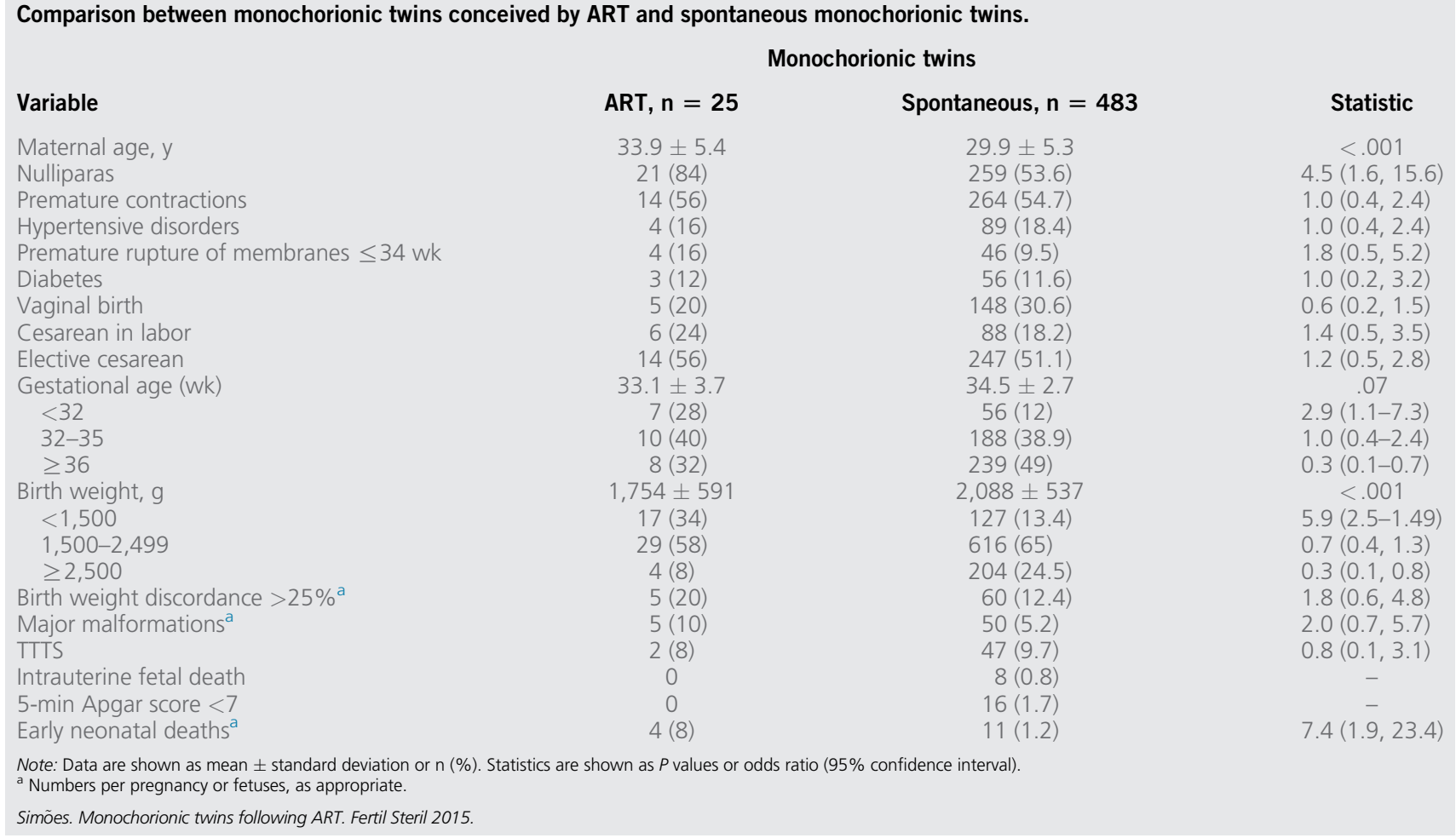


and more often nulliparous. Monochorionic twins conceived by ART were born significantly earlier and were born very preterm ( $<32$ weeks' gestation) more often compared with both control groups. This translated into significantly lower mean birth weight and more frequent very low birth weight $(<1,500 \mathrm{~g})$ babies. The frequency of discordant growth was similar as were all pregnancy complications. However, the significantly higher prematurity associated with monochorionic twins after ART led to a significantly higher early neonatal death rate.

Table 2 shows the comparison between ART twins by chorionicity. The data indicate that ART mothers were of similar age and parity irrespective of chorionicity. Otherwise, outcomes of monochorionic twins conceived by ART were significantly worse in terms of prematurity and birth weight compared with dichorionic twins conceived by ART. Although the frequencies of pregnancy complications were similar, the significantly higher prematurity associated with monochorionicity led to a significantly higher early neonatal death rate.

\section{DISCUSSION}

Although higher compared with spontaneous pregnancies, monochorionic twinning is relatively rare after ART. Hence the paucity of data related to these very high risk gestations. Nonetheless, our data indicate the two major conclusions. First and not surprisingly, monochorionic pairs have a significantly worse outcome compared with the dichorionic sets. This conclusion is not surprising because of the plethora of documented adverse outcomes related to monochorionicity irrespective of the mode of conception. The surprising finding, however, was that ART increases the already high risk of monochorionicity compared with spontaneous conception. The latter observation supports that of Mascarenhas et al. (6) but disagrees with that of Ghalili et al. (7) and Sperling et al. (8). In addition, there is no obvious explanation for the differences between the hitherto published observations.

One would assume that monochorionic twins should have similar outcomes irrespective of the mode of conception, unless there are some ill effects of assisted hatching or defective implantation. We observed similar pregnancy complications such as diabetes, hypertension, premature contractions, preterm rupture of the membranes, malformations and TTTS, and mode of delivery in both groups (Table 1); thus, it is unclear why ART is significantly associated with increased preterm birth and low birth weight rates. One might speculate that the higher rate of nulliparity in ART conception plays an important role because it has been established that nulliparity is related to significantly lower twin birth weight (9). In addition, complications related to the increased preterm birth rates might be attributed to assisted hatching or defective implantation, but, at present, the rarity of monochorionicity after ART precludes further analysis.

Our analysis does not allow drawing conclusions about the miscarriage rate associated with monochorionic

\section{TABLE 2}

Comparison between mono- and dichorionic twins conceived by ART.

\begin{tabular}{|c|c|c|c|}
\hline \multirow[b]{2}{*}{ Variable } & \multicolumn{2}{|c|}{ ART twins } & \multirow[b]{2}{*}{ Statistic } \\
\hline & Monochorionic, $n=25$ & Dichorionic, $\mathrm{n}=320$ & \\
\hline Maternal age, y & $33.9 \pm 5.4$ & $33.7 \pm 4.1$ & .86 \\
\hline Nulliparas & $21(84)$ & $278(86.9)$ & $0.7(0.3,2.8)$ \\
\hline Premature contractions & $14(56)$ & $138(43)$ & $1.6(0.7,3.9)$ \\
\hline Hypertensive disorders & $4(16)$ & $71(22.2)$ & $1.6(0.7,3.9)$ \\
\hline Premature rupture of membranes $\leq 34 \mathrm{wk}$ & $4(16)$ & $28(8.8)$ & $2.0(0.5,5.9)$ \\
\hline Diabetes & $3(12)$ & $51(15.9)$ & $0.7(0.2,2.3)$ \\
\hline Vaginal birth & $5(20)$ & $96(30)$ & $0.6(0.2,1.5)$ \\
\hline Cesarean birth in labor & $6(24)$ & (18.8) & $1.4(0.5,3.5)$ \\
\hline Elective cesarean & $14(56)$ & $163(50.9)$ & $1.2(0.5,2.9)$ \\
\hline Gestational age (wk) & $33.1 \pm 3.7$ & $35.5 \pm 2.1$ & .004 \\
\hline$<32$ & $7(28)$ & $18(5.6)$ & $6.5(2.3,17.4)$ \\
\hline $32-35$ & $10(40)$ & $94(29)$ & $1.6(0.7,3.7)$ \\
\hline$\geq 36$ & $8(32)$ & $208(65)$ & $0.3(0.1,0.6)$ \\
\hline Birth weight, g & $1,754 \pm 591$ & $2,289 \pm 454$ & $<.001$ \\
\hline$<1,500$ & $17(34)$ & $41(6.4)$ & $14.3(5.9,37)$ \\
\hline $1,500-2,499$ & $29(58)$ & $380(59.7)$ & $0.9(0.5,1.7)$ \\
\hline$\geq 2,500$ & $4(8)$ & $215(33.8)$ & $0.1(0.1,0.4)$ \\
\hline Birth weight discordance $>25 \%^{a}$ & $5(20)$ & $17(5.3)$ & $4.4(1.3,12.9)$ \\
\hline Major malformations ${ }^{a}$ & $5(10)$ & $20(3.1)$ & $3.4(1.1,9.2)$ \\
\hline TTTS & $2(8)$ & - & \\
\hline Intrauterine fetal death & 0 & $4(0.6)$ & - \\
\hline 5-min Apgar score $<7$ & 0 & $9(1.4)$ & .5 \\
\hline Early neonatal deaths ${ }^{a}$ & $4(8)$ & $6(0.9)$ & $9.0(2.2-34.2)$ \\
\hline
\end{tabular}


twinning after ART. In addition, our data set is underpowered (as all other studies) to draw conclusions related to relatively rare pregnancy complications. Yet our study points to the significant risk of monochorionicity after ART.

\section{REFERENCES}

1. Derom C, Leroy F, Vlietinck R, Fryns JP, Derom R. High frequency of iatrogenic monozygotic twins with administration of clomiphene citrate and a change in chorionicity. Fertil Steril 2006;85:755-7.

2. Blickstein I, Jones C, Keith LG. Zygotic-splitting rates after single-embryo transfers in in vitro fertilization. N Engl J Med 2003;348:2366-7.

3. Blickstein I, Keith LG. On the possible cause of monozygotic twinning: lessons from the 9-banded armadillo and from assisted reproduction. Twin Res Hum Genet 2007;10:394-9.
4. Gee RE, Dickey RP, Xiong X, Clark LS, Pridjian G. Impact of monozygotic twinning on multiple births resulting from in vitro fertilization in the United States, 2006-2010. Am J Obstet Gynecol 2014;210:468.e1-6.

5. Blickstein I. Estimation of iatrogenic monozygotic twinning rate following assisted reproduction: pitfalls and caveats. Am J Obstet Gynecol 2005;192:365-8.

6. Mascarenhas M, Kamath MS, Muthukumar K, Mangalaraj AM, Chandy A, Aleyamma T. Obstetric outcomes of monochorionic pregnancies conceived following assisted reproductive technology: a retrospective study. J Hum Reprod Sci 2014;7:119-24.

7. Ghalili A, McLennan A, Pedersen L, Kesby G, Hyett J. Outcomes of monochorionic diamniotic twin pregnancies: a comparison of assisted and spontaneous conceptions. Aust N Z J Obstet Gynaecol 2013;53:437-42.

8. Sperling L, Kiil C, Larsen LU, Qvist I, Schwartz M, Jørgensen C, et al. Naturally conceived twins with monochorionic placentation have the highest risk of fetal loss. Ultrasound Obstet Gynecol 2006;28:644-52.

9. Blickstein I. Normal and abnormal growth of multiples. Semin Neonatol 2002; 7:177-85 